\title{
Susceptibility of Propionibacterium acnes isolated from patients with acne vulgaris to zinc ascorbate and antibiotics
}

\author{
Katsuhiro linuma' \\ Norihisa Noguchi ${ }^{2}$ \\ Hidemasa Nakaminami ${ }^{2}$ \\ Masanori Sasatsu² \\ Setsuko Nishijima ${ }^{3}$ \\ Isami Tsuboi' \\ 'BML General Laboratory, Matoba, \\ Kawagoe, Saitama, ${ }^{2}$ Department of \\ Microbiology, School of Pharmacy, \\ Tokyo University of Pharmacy and \\ Life Sciences, Tokyo, ${ }^{3}$ Department of \\ Dermatology, Nishijima Skin Clinic, \\ Osaka, Japan
}

This article was published in the following Dove Press journal:

Clinical, Cosmetic and Investigational Dermatology

4 October 2011

Number of times this article has been viewed

\begin{abstract}
Purpose: The in vitro antimicrobial activity of ascorbic acid derivatives against Propionibacterium acnes was tested either alone or in combination with a variety of antimicrobial agents, and their fractional inhibitory concentration index was determined using checkerboard tests. The antimicrobial effectiveness of zinc ascorbate in the treatment of acne vulgaris, either alone or in combination with antibiotics such as clindamycin that are commonly used in Japan for the treatment of acne vulgaris, was therefore examined.
\end{abstract}

Materials and methods: The antimicrobial susceptibility of 41 strains of clindamycin-sensitive and/or clindamycin-resistant $P$. acnes isolated from acne vulgaris patients was tested, in comparison with a type strain of $P$. acnes.

Results: Zinc ascorbate showed antimicrobial activity against a type strain of $P$. acnes and its concentration $(0.064 \%)$ was sufficiently lower than the normal dose $(5 \%)$ of other ascorbic acid derivatives. Combinations of zinc ascorbate with clindamycin, erythromycin, and chloramphenicol showed an additive effect, and zinc ascorbate alone effectively inhibited the growth of all $P$. acnes including clindamycin-resistant strains.

Conclusion: The results provide novel evidence that the combination of zinc ascorbate and clindamycin is effective for acne vulgaris treatment.

Keywords: antimicrobial susceptibility, ascorbic acid derivatives, combination therapy, checkerboard test

\section{Introduction}

Propionibacterium acnes is an anaerobic, Gram-positive skin microbe that colonizes sebaceous glands and pilosebaceous follicles. ${ }^{1}$ This organism is considered to play a principal role in the development of acne vulgaris. ${ }^{1}$ Acne vulgaris is a chronic inflammatory disease characterized by typical inflammatory events, including the overproduction of sebum, abnormal desquamation of the sebaceous follicle epithelium, and P. acnes proliferation. ${ }^{1-3}$ Regarding the pathogenic factors for acne development and aggravation, ultraviolet irradiation and peroxidation of sebum lipids have been reported to activate inflammatory mediators such as reactive oxygen species. ${ }^{4}$

Ascorbic acid derivatives are some of the most widely used antioxidants for protecting the skin. ${ }^{5,6}$ The antioxidative effect of 5\% sodium ascorbyl phosphate has demonstrated efficacy in acne vulgaris. ${ }^{7}$ However, it remains unclear whether ascorbic acid derivatives have antimicrobial activity against $P$. acnes.

Many topical and systemic treatments have been proposed for acne vulgaris. ${ }^{8}$ Clindamycin and erythromycin are the most frequently used agents against $P$. acnes. ${ }^{8,9}$ On the other hand, many studies have reported the emergence of $P$. acnes with high

Correspondence: Isami Tsuboi BML General Laboratory, |36|-I, Matoba, Kawagoe-shi, Saitama 350-I I0I, Japan

Tel +8I 492323444

Fax +8I 492323135

Email i-tsuboi@bml.co.jp 
level resistance to macrolides including clindamycin because long-term antibiotic therapy is commonly used to treat acne vulgaris. ${ }^{10-12}$ In Japan, a study conducted between 1994 and 1995 revealed that macrolide-resistant strains were found in only 4\% (2/50) of $P$. acnes isolates from patients with acne vulgaris. However, they were found in $10 \%$ (5/48) of $P$. acnes isolates between 2006 and 2007, showing $P$. acnes resistance to macrolides has been increasing in Japan., ${ }^{8,9}$

Macrolide resistance in $P$. acnes is considered to be caused by mutation of the peptidyl transferase region in the domain $\mathrm{V}$ of $23 \mathrm{~S}$ rRNA, and by the target site alteration with the $23 \mathrm{~S}$ rRNA dimethylase that is encoded by $\operatorname{erm}(\mathrm{X}) .{ }^{13} \mathrm{In}$ addition, these mutations is associated with cross resistance to clindamycin. ${ }^{14}$ Therefore, the increasing prevalence of macrolide-resistant $P$. acnes, including clindamycin-resistant $P$. acnes, is a serious problem, because acne treatment by clindamycin is extremely difficult.

Recently, it has been reported that antibiotic combination enhances the therapeutic effect. ${ }^{15,16}$ In this study, the in vitro efficacy of ascorbic acid derivates against $P$. acnes alone and in combination with clindamycin, erythromycin, chloramphenicol, minocycline, or levofloxacine was examined.

\section{Material and methods Bacterial strains and drugs}

A total of $41 P$. acnes strains used in this study were isolated from patients with acne vulgaris between 2006 and 2007 in Japan, and four strains of these were clindamycin-resistant $P$. acnes with mutation of the peptidyl transferase region in the domain V of 23S rRNA gene: A2058G, or A2059G. ${ }^{9}$ The patients were given topical antibiotics instead of systemic antibiotics. The samples were cultured on modified Gifu anaerobic medium (GAM) agar (Nissui Pharmaceutical Co, Tokyo, Japan) under anaerobic conditions at $35^{\circ} \mathrm{C}$ for 72 hours. P. acnes was identified by Api 20A (bioMérieux, Marcy I'Etoile, France). ${ }^{9}$ P. acnes JCM 6473 (ATCC 11828) strain was used as the quality control strain for antimicrobial susceptibility testing. Clindamycin, erythromycin, chloramphenicol, and minocycline were purchased from SigmaAldrich (Tokyo, Japan). All other chemicals used were of the highest analytical grade.

\section{Susceptibility tests}

Susceptibility testing was performed using microbroth dilution methods, according to the criteria of the Japanese Society of Chemotherapy. ${ }^{17,18}$ The samples were cultured in GAM broth (Nissui Pharmaceutical Co) and adjusted to a
McFarland standard number 1. A dilute bacterial suspension was used to inoculate wells of a 96-well microplate, each well containing a different concentration of the drug being tested. Double dilutions of the drugs were prepared. The concentrations of the drugs in the GAM broth ranged from 0.06 to $128 \mu \mathrm{g} / \mathrm{mL}$ (antimicrobial agents) or 1.25 to $1280 \mu \mathrm{g} / \mathrm{mL}$ (ascorbic acid derivatives), and a final concentration of $10^{5}$ colony-forming units (CFU) of test bacteria per well was added to each dilution. The plates were incubated in an anaerobic gas-generating pouch (Anaero Pack System; Mitsubishi Gas Chemical Co, Tokyo, Japan) at $35^{\circ} \mathrm{C}$ for 48 hours. After the positive control lacking the antimicrobial agent demonstrated good growth, the minimum inhibitory concentration (MIC) for each antibiotic was defined as the lowest concentration of antibiotic required to inhibit bacterial growth, indicated by the absence of turbidity.

\section{Fractional inhibitory concentration (FIC) index}

The efficacy of a combination of zinc ascorbate and antimicrobial agents against $P$. acnes was determined by checkerboard tests using microbroth dilution methods. ${ }^{19}$ Fractional inhibitory concentration (FIC) indices were calculated using the following formula: FIC index $=($ MIC of drug A in combination/MIC of drug A alone) + (MIC of drug B in combination/MIC of drug B alone). ${ }^{20} \mathrm{An}$ index of $<0.5$ was considered as synergism; that $<1.0$ but $>0.5$ as additive action; and that $>2.0$ as antagonism. The samples were adjusted to the McFarland standard number 1 and a final concentration of $10^{5} \mathrm{CFU} /$ well of test bacteria. The MICs of the combinations were determined after incubation at $35^{\circ} \mathrm{C}$ for 48 hours.

\section{Results}

\section{The antibiotic susceptibility of $P$. acnes to ascorbic acid derivatives}

The antibiotic susceptibility of $P$. acnes JCM 6473 to ascorbic acid derivatives zinc ascorbate, magnesium ascorbyl phosphate, and sodium ascorbyl phosphate was investigated (Table 1). The MIC of zinc ascorbate was $640 \mu \mathrm{g} / \mathrm{mL}$, whereas the MICs of ascorbic acid, magnesium ascorbyl phosphate, and sodium ascorbyl phosphate were $\geq 1280 \mu \mathrm{g} / \mathrm{mL}$. The normal dose of ascorbic acid derivatives is $5 \%(50 \mathrm{mg} / \mathrm{mL}){ }^{7}$ These results indicate that zinc ascorbate sufficiently inhibits the growth of $P$. acnes in the normal dose. Thus, zinc ascorbate was used in further experiments. 
Table I Susceptibility of Propionibacterium acnes JCM 6473 to ascorbic acid derivatives

\begin{tabular}{ll}
\hline Ascorbic acid derivates & MIC \\
\hline Ascorbic acid & $\geq 1280$ \\
Zinc ascorbate & 640 \\
Magnesium ascorbyl phosphate & $\geq 1280$ \\
Sodium ascorbyl phosphate & $\geq 1280$ \\
\hline
\end{tabular}

Abbreviation: MIC, minimum inhibitory concentration $(\mu \mathrm{g} / \mathrm{mL})$.

\section{Combined effect of zinc ascorbate and various antimicrobial agents against $P$. acnes}

To study the antimicrobial effects of combinations of zinc ascorbate and antimicrobial agents such as clindamycin, erythromycin, chloramphenicol, minocycline, and levofloxacin, the FIC index was determined by checkerboard tests (Table 2). In combinations of zinc ascorbate with clindamycin, erythromycin, or chloramphenicol, the MIC of zinc ascorbate reduced, and the FIC indices ranged from 0.625 to 0.75 . The values exhibited an additive effect against $P$. acnes JCM 6473. Conversely, the FIC indices for the combinations of zinc ascorbate with minocycline or levofloxacin showed no effect.

Clindamycin is approved and commonly used in Japan for the treatment of acne vulgaris. ${ }^{8,9}$ Thus, the antimicrobial effect of zinc ascorbate combined with clindamycin for 37 strains of clindamycin-sensitive $P$. acnes isolated from patients with acne vulgaris was investigated. As shown in Table 3, the MIC range of zinc ascorbate was approximately twofold lower in combination with clindamycin compared with that of zinc ascorbate and clindamycin alone. The average FIC index was 0.84 , exhibiting an additive effect.

Table 2 Combined effects of zinc ascorbate and antimicrobial agents against Propionibacterium acnes JCM 6473

\begin{tabular}{lll}
\hline Drug & MIC (FIC index) & Interaction \\
\hline Zinc ascorbate & 640 & \\
Clindamycin & 0.03 & \\
Erythromycin & 0.13 & \\
Chlorlamphenicol & 2 & \\
Minocycline & 0.25 & \\
Levofloxacin & 0.5 & Additive \\
Zinc ascorbate/Clindamycin & $80 / 0.02(0.63)$ & Additive \\
Zinc ascorbate/Erythromycin & $160 / 0.06(0.75)$ & Additive \\
Zinc ascorbate/Chloramphenicol & $160 / 1(0.75)$ & Antagonism \\
Zinc ascorbate/Minocycline & $640 / 0.25(2)$ & Antagonism \\
Zinc ascorbate/Levofloxacin & $640 / 0.5(2)$ & \\
\hline
\end{tabular}

Note: The interaction was defined as synergistic if the FIC index was $<0.5$, additive if the FIC index was between 0.5 and I.0, antagonistic if the FIC index $\geq 2$.

Abbreviations: MIC, minimum inhibitory concentration $(\mu \mathrm{g} / \mathrm{mL})$; FIC, fractional inhibitory concentration.
Combined effect of zinc ascorbate and various antimicrobial agents against clindamycin-resistant $P$. acnes isolated from patients with acne vulgaris

Clindamycin-resistant $P$. acnes with mutation of the peptidyl transferase region in the domain $\mathrm{V}$ of $23 \mathrm{~S}$ rRNA has been increasing in Japan. ${ }^{9}$ The antimicrobial effect of zinc ascorbate combined with clindamycin, erythromycin, and chloramphenicol for four strains (PA001-PA004) of clindamycin-resistant $P$. acnes with a transition of adenine to guanine at the position of 2058 (A2058G: PA001-PA003) or 2059 (A2059G: PA004) was investigated. As shown in Table 4, zinc ascorbate was equally effective at inhibiting the growth of clindamycin-resistant $P$. acnes strains (MIC $640 \mu \mathrm{g} / \mathrm{mL}$ ). In combinations of zinc ascorbate with clindamycin, erythromycin, and chloramphenicol, the MIC of zinc ascorbate reduced (MIC range: $160-320 \mu \mathrm{g} / \mathrm{mL}$ ). In addition, the FIC index of PA004 strain with clindamycinresistant $P$. acnes was 0.75 , exhibiting an additive effect.

\section{Discussion}

In the microbroth dilution methods, according to the criteria of the Japanese Society of Chemotherapy, a twofold serial dilution series is used to determine the effectiveness of the test drug, with $1 \mu \mathrm{g} / \mathrm{mL}$ as the primary dilution. ${ }^{17,18}$ In this study, the concentrations of the drugs ranged from 0.06 to $128 \mu \mathrm{g} / \mathrm{mL}$. However, ascorbic acid derivatives showed no effect in this concentration. It was confirmed that MIC of zinc was $1280 \mu \mathrm{g} / \mathrm{mL}$ (data not shown), which is similar to that reported by Fluhr et al. ${ }^{21}$ Therefore, the concentrations of ascorbic acid derivatives ranged from 1.25 to $1280 \mu \mathrm{g} / \mathrm{mL}$.

Five percent of sodium ascorbyl phosphate has been reported to reduce acne lesions by $20.14 \%$ and $48.82 \%$ within 4 and 8 weeks, respectively. ${ }^{7}$ In addition, topical $(1.2 \%)$ and systemic $(30 \mathrm{mg})$ zinc have been reported to show efficacy in the treatment of acne vulgaris. ${ }^{21,22}$ In the present study, the MIC $(640 \mu \mathrm{g} / \mathrm{mL})$ of zinc ascorbate against $P$. acnes JCM 6473 corresponded to $0.064 \%$, and the MIC value was lower than other ascorbic acid derivatives (MIC $\geq 1280 \mu \mathrm{g} / \mathrm{mL}$ ). Although further experiments are needed to examine the drug kinetics and the stability of zinc ascorbate, it is expected that the calculating concentration of zinc ascorbate would be $2800 \mu \mathrm{g} / \mathrm{mL}$ when it is applied on the skin $\left(4 \mathrm{mg} / \mathrm{cm}^{2}\right)$ as an external preparation. ${ }^{23}$ These results suggest a possibility that zinc ascorbate (MIC 
Table 3 Combined effect of zinc ascorbate and clindamycin against 37 strains of Propionibacterium acnes isolated from patients with acne vulgaris

\begin{tabular}{llllll}
\hline Drug & MIC ranges $\left(\mathbf{M I C}_{90}\right)$ & & FIC & Interaction \\
\cline { 2 - 3 } \cline { 5 - 6 } & Alone & Combined & & Range & Average \\
\hline Clindamycin & $0.13-2$ & $0.06-1$ & $0.63-1$ & 0.84 \\
Zinc ascorbate & $(2)$ & $(1)$ & & \\
& $160-640$ & $80-320$ & & \\
\hline
\end{tabular}

Abbreviations: MIC, minimum inhibitory concentration $(\mu \mathrm{g} / \mathrm{mL})$; FIC, fractional inhibitory concentration; $\mathrm{MIC}_{90}$, concentration of drugs which inhibited growth in $90 \%$ of the strains tested.

$640 \mu \mathrm{g} / \mathrm{mL}$ ) sufficiently inhibits the growth of $P$. acnes in the concentration that is lower than the normal dose of other ascorbic acid derivatives and zinc.

Choi et $\mathrm{al}^{24}$ reported that the antimicrobial mechanism involves the capability of zinc ions to inhibit glycolysis of microorganisms by oxidizing thiol groups in essential glycolytic enzymes, therefore, the antimicrobial mechanism of zinc ascorbate may be similar to that of zinc. However, a combined effect of zinc ascorbate and minocycline or levofloxacin was not proved. Clindamycin, erythromycin, and chloramphenicol inhibit the protein synthesis of bacteria by binding to the $50 \mathrm{~S}$ subunit of the bacterial ribosome. ${ }^{25}$ In contrast, the binding sites of minocycline and levofloxacin are on the $30 \mathrm{~S}$ subunit of the ribosome and on DNA topoisomerase IV, respectively. ${ }^{26,27}$ Thus, the difference in antibacterial mechanism may be related to the combined effect of each of these. In addition, it is possible that minocycline and levofloxacin chelate with metal ions, including zinc, ${ }^{28,29}$ resulting in reduced antimicrobial activity. Further experiments are needed to clarify the mechanism of zinc ascorbate against $P$. acnes.

Macrolides are used not only as antimicrobial agents but also as anti-inflammatory agents, and are the most commonly used to treat acne vulgaris. ${ }^{9}$ Clindamycin shows a similar effect to macrolides and is the most popular topical antibacterial for acne vulgaris in Japan. However, clindamycin-resistant P. acnes strains have been increasing in Japan. ${ }^{8,9}$ An increase of clindamycin-resistant $P$. acnes including macrolides and an epidemic of the multiple-drug-resistant $P$. acnes can be expected in the future. It is reported that combination therapy with antimicrobial agents and other agents such as the external retinoids and benzoyl peroxide is effective in preventing the emergence of the antibiotic-resistant strains of $P$. acnes. ${ }^{15,16}$ In the present study, it was found that zinc ascorbate inhibited the growth of the clindamycin-resistant P. acnes strains (MIC $640 \mu \mathrm{g} / \mathrm{mL}$ ). In addition, although the FIC of zinc ascorbate in combination with clindamycin was unable to be calculated because the MIC of clindamycin alone and in combination was $\geq 128 \mu \mathrm{g} / \mathrm{mL}$ (3/4 strains), it reduced the MIC of zinc ascorbate against all strains. Moreover, the FIC index of PA004 strain with clindamycinresistant $P$. acnes was 0.75 , exhibiting an additive effect. These results strongly suggest that the combination of zinc ascorbate and clindamycin will be useful for preventing the emergence of clindamycin-resistant $P$. acnes and for treating acne vulgaris.

Table 4 Combined effect of zinc ascorbate and antimicrobial agents against four strains of macrolides-resistant Propionibacterium acnes isolated from patients with acne vulgaris

\begin{tabular}{|c|c|c|c|c|c|c|c|}
\hline \multirow{2}{*}{$\begin{array}{l}\text { Strain } \\
\text { no. }\end{array}$} & \multicolumn{7}{|c|}{ MIC (FIC index) } \\
\hline & $\mathbf{A Z n}$ & CLDM & EM & CP & AZn/CLDM & AZn/EM & $\mathrm{AZn} / \mathrm{CP}$ \\
\hline PA00I & 640 & $\geq 128$ & $\geq 128$ & 2 & $\begin{array}{l}160 / \geq 128 \\
(N C)\end{array}$ & $\begin{array}{l}\mid 60 / \geq 128 \\
(N C)\end{array}$ & $\begin{array}{l}320 / 1 \\
(1)\end{array}$ \\
\hline PA002 & 640 & $\geq 128$ & $\geq 128$ & 2 & $\begin{array}{l}320 / \geq 128 \\
(N C)\end{array}$ & $\begin{array}{l}320 / \geq 128 \\
(N C)\end{array}$ & $\begin{array}{l}320 / 1 \\
(1)\end{array}$ \\
\hline PA003 & 640 & $\geq 128$ & $\geq 128$ & I & $\begin{array}{l}320 / \geq 128 \\
(N C)\end{array}$ & $\begin{array}{l}\mid 60 / \geq 128 \\
(N C)\end{array}$ & $\begin{array}{l}160 / 0.5 \\
(0.75)\end{array}$ \\
\hline PA004 & 640 & 64 & $\geq 128$ & I & $\begin{array}{l}160 / 32 \\
(0.75)\end{array}$ & $\begin{array}{l}320 / \geq 128 \\
(\mathrm{NC})\end{array}$ & $\begin{array}{l}160 / 0.5 \\
(0.75)\end{array}$ \\
\hline
\end{tabular}

Abbreviations: MIC, minimum inhibitory concentration ( $\mu \mathrm{g} / \mathrm{mL})$; FIC, fractional inhibitory concentration; PA, Propionibacterium acnes; AZn, zinc ascorbate; CLDM, clindamycin; EM, erythromycin; CP, chloramphenicol; NC, not calculated. 


\section{Conclusion}

The results of this study provide novel evidence that zinc ascorbate inhibits the growth of $P$. acnes, and its concentration $(0.064 \%)$ is sufficiently lower than the normal dose $(5 \%)$ of other ascorbic acid derivatives. In addition, zinc ascorbate shows an additive antimicrobial effect in vitro in combination with clindamycin, erythromycin, and chloramphenicol against clindamycin-sensitive and/or clindamycin-resistant $P$. acnes. These findings may provide novel insights into acne therapy. Recently, combination therapy with benzoyl peroxide and clindamycin has become common worldwide. Further experiments are needed to compare zinc ascorbate plus clindamycin and benzyol peroxide plus clindamycin.

\section{Disclosure}

The authors report no conflicts of interest in this work.

\section{References}

1. Bojar RA, Holland KT. Acne and Propionibacterium acnes. Clin Dermatol. 2004;22:357-379.

2. Iinuma K, Sato T, Akimoto N, et al. Involvement of Propionibacterium acnes in the augmentation of lipogenesis in hamster sebaceous glands in vivo and in vitro. $J$ Invest Dermatol. 2009;129:2113-2119.

3. Farrar MD, Ingam E. Acne: Inflammation. Clin Dermatol. 2004;22: 380-384.

4. Akamatsu H, Horio T, Hattori K. Increased hydrogen peroxide generation by neutrophils from patients with acne inflammation. Int $J$ Dermatol. 2003;42:366-369.

5. Pinnell S, Madey D. The benefits of topical Vitamin C (L-ascorbic acid) for skin care and UV-protection. J Appl Cosmetol. 1999;18:126-134.

6. Burgess C. Topical vitamins. J Drugs Dermatol. 2008;7:2-6.

7. Ruamrak C, Lourith N, Natakankitkul S. Comparison of clinical effcacies of sodium ascorbyl phosphate, retinol and their combination in acne treatment. Int J Cosmet Sci. 2009;31:41-46.

8. Nishijima S, Kurokawa I, Kawabata S. Sensitivity of Propionibacterium acnes isolated from acne patients: comparative study of antimicrobial agents. J Int Med Res. 1996;24:473-477.

9. Ishida N, Nakaminami H, Noguchi N, Kurokawa I, Nishijima S, Sasatsu M. Antimicrobial susceptibilities of Propionibacterium acnes isolated from patients with acne vulgaris. Microbiol Immunol. 2008;52: $621-624$.

10. Ross JI, Snelling AM, Eady EA, et al. Phenotypic and genotypic characterization of antibiotic-resistant Propionibacterium acnes isolated from acne patients attending dermatology clinics in Europe, the USA, Japan and Australia. Br J Dermatol. 2001;144:339-346.

11. Eady EA, Gloor M, Leyden JJ. Propionibacterium acnes resistance: a worldwide problem. Dermatology. 2003;206:54-56.
12. Heller S, Kellenberger L, Leyden JJ. Antipropionibacterial activity of BAL19403, a novel macrolide antibiotic. Antimicrob Agents Chemother. 2007;51:1956-1961.

13. Ross JL, Snelling AM, Carnegie E, et al. Antibiotic-resistant acne: lessons from Europe. Br J Dermatol. 2003;148:467-478.

14. Ross JL, Eady EA, Carnegie E, Cove JH. Detection of transpospn Tn5432-mediated macrolide-lincosamide-streptogramin B (MLSB) resistance in cutaneous propionibacteria from six European cities. J Antimicrob Chemother. 2002;49:165-168

15. Gollnick H, Cunliffe W, Berson D, et al. Management of acne: a report from a Global Alliance to Improve Outcomes in Acne. J Am Acad Dermatol. 2003;49:S1-S37.

16. Dreno B. Topical Antibacterial Therapy for Acne Vulgaris. Drugs. 2004;64:2389-2397.

17. Japan Society of Chemotherapy. Method for the determination of minimum inhibitory concentration (MIC) of anaerobic bacteria by agar dilution method. Chemotherapy. 1979;27:559-560.

18. Japan Society of Chemotherapy. Microbroth dilution methods for determination of the minimum inhibitory concentrations in bacteria Chemotherapy. 1993;41:183-189.

19. Horrevorts AM, de Ridder CM, Poot MC, et al. Chequerboard titrations: the influence of the composition of serial dilutions of antibiotics on the fractional inhibitory concentration index and fractional bactericidal concentration index. J Antimicrob Chemother. 1987;19: 119-125.

20. Hewlett PS. Measurement of the potencies of drug mixtures. Biometrics. 1969;25:477-487.

21. Fluhr JW, Bösch B, Gloor M, Höffler U. In-vitro and in-vivo efficacy of zinc acetate against propionibacterium alone and in combination with erythromycin. Zentralbl Bakteriol. 1999;289:445-456.

22. Dreno B, Moyse D, Alirezai M, et al. Multicenter randomized comoarative double-bind controlled clinical trial of the safety and efficacy of zinc gluconate versus minocycline hydrochloride in the treatment of inflammatory acne vulgaris. Dermatology. 2001;203:135-140.

23. Long CC, Finlay AY. The finger-tip unit - a new practical measure Clin Exp Dermatol. 1991;16:444-447.

24. Choi EK, Lee HH, Kang MS, et al. Potentiation of bacterial killing activity of zinc chloride by pyrrolidine dithiocarbamate. J Microbiol. 2010;48:40-43.

25. Vannuffel P, Cocoto C. Mechanism of action of streptogramins and macrolides. Drugs. 1996;51:20-30.

26. Griffin MO, Fricovsky F, Ceballos G, Villarreal F. Tetracyclines: a pleitropic family of compounds with promising therapeutic properties. Review of the literature. Am J Physiol Cell Physiol. 2010;299. 539-548.

27. Tanaka M, Onodera Y, Uchida Y, Sato K, Hayakawa I. Inhibitory activities of quinolones against DNA gyrase and topoisomerase IV purified from Staphylococcus aureus. Antimicrob Agents Chemother. 1997;41:2362-2366

28. Brion M, Lambs L, Berthon G. Metal ion-tetracycline interactions in biological fluids. Part 5. Formation of zinc complexes with tetracycline and some of its derivatives and assessment of their biological significance. Agents Actions. 1985;17:229-242.

29. Stein GE. Drug interactions with fluoroquinolones. Am J Med. 1991;91: 81S-86S.
Clinical, Cosmetic and Investigational Dermatology

\section{Publish your work in this journal}

Clinical, Cosmetic and Investigational Dermatology is an international, peer-reviewed, open access, online journal that focuses on the latest clinical and experimental research in all aspects of skin disease and cosmetic interventions. All areas of dermatology will be covered; contributions will be welcomed from all clinicians and

\section{Dovepress}

basic science researchers globally. This journal is indexed on CAS The manuscript management system is completely online and includes a very quick and fair peer-review system, which is all easy to use. Visit http://www.dovepress.com/testimonials.php to read real quotes from published authors. 